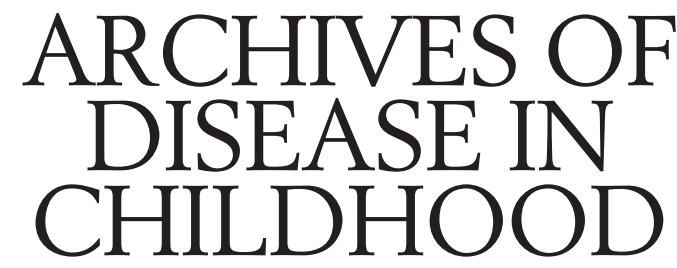

The Fournal of the Royal College of Paediatrics and Child Health

\title{
Annotations
}

\section{Echocardiography on the neonatal unit: a job for the neonatologist or the cardiologist?}

Over recent years echocardiography has changed from a purely research tool to an essential part of adequate management of the critically ill newborn. In the hypotensive or shocked infant, there is no better way to find a treatable underlying cause of their circulatory failure such as myocardial dysfunction, a large ductal shunt, pericardial effusion, or hypertrophic cardiomyopathy in infants of mothers with diabetes. A large ductal shunt sufficient to cause severe reduction in effective cardiac output and even cardiorespiratory collapse after extubation can be clinically silent and may only be revealed by echocardiography. ${ }^{1}$ Similarly, septic vegetations or thrombi on valves or central lines can only be seen this way, and it is feasible to assess pulmonary arterial pressure non-invasively in most ill newborns.

Therefore, someone needs to be available 24 hours a day in all neonatal units to do echocardiography. There are about 60 paediatric cardiologists in the UK, about one per million of the population; this is simply not enough to provide a 24 hour service for all neonatal units, many of which are long distances from big cities. Some units outside the UK have echocardiography technicians based and trained within the paediatric cardiac unit who provide a useful service - for example, screening on the third day of life for ductal shunting, but even they are not usually available outside normal working hours.

\section{Should neonatologists be doing the scans?}

To date, I have run five echocardiography courses specifically designed for neonatologists. All have been oversubscribed and other recent courses elsewhere in the UK have been equally popular. Neonatologists feel the need to learn echocardiography and most trainees leave the courses with more rather than less respect for the technique. Recognising this, I approached the committee of the British Society of Echocardiography, of which I am a member, to ask if they could help to organise national guidelines and set standards for such courses; they have taken on the role of accreditation of both echocardiography courses and trainees. The idea was firmly rejected-the committee did not feel that such courses were desirable. Many paediatric cardiologists are uncomfortable with neonatologists doing echocardiography because they feel they may miss or wrongly diagnose congenital heart disease (CHD). How justified are they in believing this?

During a research project using echocardiography in a neonatal unit, ${ }^{2} 33$ babies being treated for persistent hypoxaemia were assessed echocardiographically: one was found to have transposed great arteries and another had severe hypertrophic cardiomyopathy. Some cyanotic CHD can be difficult to diagnose echocardiographically (such as anomalous pulmonary venous drainage) and these infants must be seen by a paediatric cardiologist. ${ }^{4}$ However, among 60 infants examined using echocardiography to assess ductal shunting, none had CHD. One infant from another institution was referred with coarctation having been given indomethacin without previously being evaluated echocardiographically. Echocardiographic evaluation of ductal shunting is necessary before treatment, relatively easy to learn, and aortic coarctation can be excluded in this group before giving indomethacin by confirming that pure left to right ductal shunting is present using Doppler in association with good foot pulses.

As the British Society of Echocardiography will not help us, I would like to begin a discussion on this subject by proposing who should be trained, how they should be trained, and what should and should not be expected of a trainee. This is based on my experience as a paediatric cardiologist with experience in neonatology, a background of research into neonatal haemodynamics, and experience in teaching echocardiography to neonatologists.

\section{Who should learn echocardiography on the neonatal unit?}

Each unit should have one consultant with special skills in echocardiography. Some trainees in neonatology may usefully elect to spend a year or more within a paediatric cardiac centre to train in echocardiography and $\mathrm{CHD}-\mathrm{a}$ handful of neonatologists in post in the UK have already done this.

\section{How should they be trained?}

Each trainee should attend a course in basic echocardiography and paediatric echocardiography, currently available from the British Society of Echocardiography, and/or a specialist course in echocardiography for the neonatologist. The neonatologist echocardiographer should have ongoing, regular, and documented contact with a local paediatric cardiac unit allowing continued exposure to echocardiography in CHD and allowing for specialist audit. Some form of certification of competence needs to be organised through a body as yet unspecified. 
Which infants should be referred to the paediatric cardiologist?

- Those with clinically suspected congenital heart disease

- Those ventilated for severe persistent hypoxaemia, particularly if extracorporeal membrane oxygenation is being considered

- Those in whom an adequate echocardiogram is not obtained by the neonatologist or where echocardiography reveals previously unsuspected CHD.

\section{Which infants can reasonably be assessed by a} trained neonatologist echocardiographer?

- The hypotensive or shocked newborn in the first few hours of life without clinical evidence of congenital heart disease

- Those requiring assessment of ductal and/or interatrial shunting

- Those with a central line to assess its position or to exclude vegetation or thrombus

- Those in whom pulmonary arterial pressure or cardiac output needs to be assessed (once CHD has been excluded by a paediatric cardiologist in infants with persistent hypoxaemia).

\section{Conclusions}

Echocardiography is an essential part of modern neonatal intensive care. Its use should not be limited to cardiologists in the diagnosis and assessment of CHD but

\section{Licensing of medicines}

The Medicines Act 1968 requires that all medicines manufactured or marketed in the UK have been authorised by the licensing authority, the Medicines Control Agency. The aim of the licensing system is to ensure that medicines are examined for efficacy, safety, and quality. Pharmaceutical companies apply for a product licence for a particular drug, and in their submission they include the indication, dose, route of administration, and age group of patient for which this applies. The licensing arrangements constrain pharmaceutical companies in that they cannot promote either an unlicensed product or a licensed product for an unlicensed indication. Doctors, however, are not restricted to prescribing licensed medicines or for licensed indications.

\section{Drug toxicity and licensing}

The Medicines Act was a response to some of the major cases of drug toxicity that occurred in the late 1950s and early 1960s. Two of these resulted in significant morbidity and mortality for the developing fetus and the newborn infant: phocomelia caused by thalidomide ${ }^{12}$ and the grey baby syndrome caused by chloramphenicol. ${ }^{3}$ It is ironic that, despite drug toxicity occurring in this age group, neonates, infants, and children receive medicines that have not been through the licensing system. Many of the medicines given to children in hospitals either do not have a product license for use in children and hence are used off label (outside the terms of the product licence) or are simply not licensed at all for use in their current formulation. ${ }^{4}$ Recent studies suggest that up to $40 \%$ of children in hospital receive "unlicensed" medicines ${ }^{5}$ and this is of concern to children, their parents, health professionals, the regulatory authorities, and, more recently, politicians. ${ }^{6}$ should be extended to the routine care of critically ill neonates. As with any investigative tool, echocardiography should be used in combination with clinical acumen and not as a replacement. Paediatric cardiologists should not be worried about neonatologists learning echocardiography, rather they should encourage, support and supervise them-live video links may even help to avoid unnecessary transfer to cardiac centres of cyanotic infants without CHD. Being involved in establishing guidelines for the safe practice of neonatal echocardiography is surely better than ignoring it.

\section{J R SKINNER}

\author{
Department of Cardiology, \\ Bristol Children's Hospital, \\ St Michael's Hill, \\ Bristol BS2 8Bf, UK \\ email:jonathanskinner@compuserve.com
}

1 Skinner JR, Hunter S, Hey EN. Cardiorespiratory collapse and the silent ductus [abstract]. Klinische Padiatrie 1991;203:52.

2 Skinner JR, Hey EN, Hunter S. Echocardiography in the neonatal unit-a job for the cardiologist or the neonatologist? [abstract] British Paediatric job for the cardiologist or the neonatologis
Association meeting, York, March 1995.

3 Skinner JR, Hunter S, Hey EN. Haemodynamic features at presentation in persistent pulmonary hypertension of the newborn and outcome. Arch Dis Child 1996;74:F26-32.

4 Linday LA, Ehlers KH, O'Loughlin JE, LaGamma EF, Engle MA. Noninvasive diagnosis of persistent fetal circulation versus congenital heart defects. Am F Cardiol 1983;52:847-51.
Pharmaceutical companies have been reluctant to seek licences for use of their products in children for a variety of reasons - the practical difficulties in organising clinical trials, the lack of a major financial incentive as the paediatric market is significantly less than that for adults, concerns about drug toxicity, and inexperience in conducting clinical trials in children. ${ }^{7}$ It is often more difficult to organise clinical trials in children than in adults; however, adequate clinical trials can be carried out in this age group $^{8-10}$ and the fear of carrying out such trials is usually far greater than the practical difficulties involved. Children may be at greater risk for certain toxic effects and this has been highlighted by the hepatotoxicity caused by sodium valproate, which resulted in the death of more than 100 patients (most of whom were children), ${ }^{11}$ and the development of Reye's syndrome following the use of salicylates. $^{12}$

\section{The need to study drugs in children}

Several problems have illustrated that dosing regimens derived from adult studies cannot be extrapolated to infants and children. The importance of understanding drug metabolism in relation to development is illustrated by the toxicity of thalidomide, chloramphenicol, and sodium valproate. Thalidomide itself is not teratogenic but its dicarboxylic acid metabolite is teratogenic. ${ }^{13}$ This metabolite, however, cannot cross the placenta and the teratogenicity of thalidomide is because of formation of the toxic metabolite by the fetus itself. An understanding of fetal metabolism is important in relation to both understanding and hopefully preventing further cases of teratogenicity. The initial dosage regimen for chloramphenicol was based on studies in adults; however, the 
metabolism of chloramphenicol is impaired in neonates and therefore significantly lower doses are required to prevent toxicity. ${ }^{14}$ Drug toxicity is not restricted to the fetus and the newborn infant; sodium valproate hepatotoxicity is thought to be related to enhanced omega oxidation. ${ }^{15}$ This pathway is enhanced by polypharmacy and certain metabolic diseases, it also appears to be enhanced in children younger than 3 years. These examples illustrate the importance of increasing our knowledge of drug metabolism in children to minimise toxicity while ensuring efficacy.

\section{Initiatives}

Medicines for adults are evaluated to ensure that they meet acceptable standards of safety, quality, and efficacy, whereas in children they are often not evaluated. This unsatisfactory situation resulted in the setting up of a joint working party between the (then) British Paediatric Association and the Association of the British Pharmaceutical Industry, and the publication of a joint report on the licensing of medicines for children in May $1996 .{ }^{16}$ At the same time there was discussion in the Europe Community regarding the guidance on clinical investigation of medicinal products in children. After consultation involving health professionals throughout the European Community a new guidance has been issued (March 1997), which came into force in September 1997. ${ }^{17}$ The new guidance is a major step forward because of its emphasis on the need for product testing in children. It divides medicinal products into four categories with the following recommendations:

(1) Diseases affecting children exclusively-trials of medicinal products in children may start before any adult human exposure

(2) Diseases that mainly affect children, are of particular gravity in children, or have a different natural history in children-clinical trials in children are needed at an early stage in clinical development following demonstration of safety and reasonable (phase I and II) evidence of efficacy in adults

(3) Diseases occurring in adults and children for which there is currently no treatment - as point 2

(4) Diseases occurring in adults and children for which treatment exists-clinical trials in children should usually follow completion of adult phase III trials.

This is a sensible approach that should encourage the pharmaceutical industry to concentrate on clinical trials for conditions in children where there is either no or inadequate treatment at present.

\section{Response}

The new guidance also encourages pharmaceutical companies to carry out trials in children where the product is likely to be used in children. In the past there was no pres- sure on the pharmaceutical company to carry out trials in children. The recent report by the House of Commons health committee on the specific health needs of children and young people confirmed that the present system was unacceptable. ${ }^{6}$ The Royal College of Paediatrics and Child Health through its medicines committee has shown that it is keen to cooperate with both the pharmaceutical industry and the regulatory authorities. The Medicines Control Agency in the UK has played a leading role in the development of the new guidance for Europe. We hope that pharmaceutical companies respond to the challenge of ensuring that medicines used in children are evaluated scientifically.

I CHOONARA

Academic Division of Child Health,

Derbyshire Children's Hospital

Uttoxeter Road

Derby DE22 3NE, UK

J DUNNE

Medicines Control Agency,

Market Towers,

1 Nine Elms Lane,

London SW8 $5 N Q, U K$

1 McBride WD. Thalidomide and congenital abnormalities. Lancet 1961;ii: 1358.

2 Lenz W. Thalidomide and congenital abnormalities. Lancet 1967;i:45.

3 Sutherland JM. Fatal cardiovascular collapse of infants receiving large amounts of chloramphenicol. Am f Dis Child 1959;97:761-7.

4 Turner S, Nunn AJ, Choonara I. Unlicensed drug use in children in the UK. Paediatric and Perinatal Drug Therapy 1997;1:52-5.

5 Turner S, Gill A, Nunn T, Hewitt B, Choonara I. Use of "off-label" and unlicensed drugs in paediatric intensive care unit. Lancet 1996;347:549-50.

6 House of Commons Health Committee. 2nd report. The special health needs of children and young people. London: HMSO, 1997.

7 Kearns GL, Reed MD. Immediate action needed to improve labelling of prescription drugs for pediatric patients. Ann Pharmacother 1997;31: 249-51.

8 Cornelissen EAM, Kollee LAA, De Abreu RA, et al. Effects of oral and intramuscular vitamin $\mathrm{K}$ prophylaxis on vitamin $\mathrm{K} 1$, PIVKA-II and clotting factors in breast fed infants. Arch Dis Child 1992;67:1250-4.

9 Benini F, Johnston C, Faucher D, Aranda J V. Topical anaesthesia during circumcision in newborn infants. $\mathcal{F} A M A$ 1993;270:850-3.

10 Parkinson L, Hughes J, Gill A, Billingham I, Ratcliffe J, Choonara I. A randomised controlled trial of sedation in the critically ill. Paediatr Anaesth 1997;7:405-10.

11 Menander KA. Valproic acid and hepatic fatalities: a retrospective review. Neurology 1987;37:379-85.

12 Woodall DF. Reye's syndrome and salicylate use. Pediatrics 1980;66:859-64.

13 Aranda JV, Stern L. Clinical aspects of developmental pharmacology and toxicology. Pharmacol Ther 1983;20:1-51.

14 Weiss CF, Glazko AJ, Weston JK. Chloramphenicol in the newborn infant. N Engl F Med 1960;262:787-94.

15 Fisher E, Siemes H, Pund R, Wittfoht W, Nau H. Valproate metabolites in serum and urine during antiepileptic therapy in children with infantile spasms: abnormal metabolite pattern associated with reversible hepatotoxicity. Epiepsia 1992;33:165-71.

16 Licensing medicines for children. London: British Paediatric Association, May 1996.

17 Note for guidance on clinical investigation of medicinal products in children. London: Medicines Control Agency, 1997

\section{Epidemiology of head injury}

Head injury is recognised as a major public health problem that is a frequent cause of death and disability in young people and makes considerable demands on health services. Epidemiological data are required to initiate appropriate preventive measures and to plan necessary services. However, reliable statistics are difficult to extract from routinely collected data.

International statistics for accidental deaths and road accident deaths do not identify head injuries, but they do indicate differences in accident rates between countries and over time. For example, road traffic accident (RTA) deaths are more than twice as frequent in France, Australia, and the USA as in the UK or the Netherlands, but in developed countries they are steadily decreasing each year. ${ }^{1}$ In developing countries accident rates are increasing as traffic increases, and they greatly exceed those of developed countries. Asked about the main health hazard of the next decade a Chinese professor of public health 
replied "the motorcycle". Head injuries account for one quarter to one third of all accidental deaths, and for two thirds of trauma deaths in hospital. They are also the main cause of lifelong disability after trauma.

While the codes of the International Classification of Diseases do allow some estimates of the frequency of head injuries from routine statistics on deaths and hospital discharges, the 10 codes that cover head injury are variably applied; moreover, multiple injuries and transfers after first admission make estimates inaccurate. Injury severity scores used for trauma in general have been shown to be very inaccurate when applied to head injuries. Although people who present to hospital but are not admitted are a major part of the head injury problem for hospitals, no routine statistics are published for accident and emergency departments. Data published from clinical series are mostly from neurosurgeons whose selection criteria for transfer vary widely and whose population base can seldom be defined, making these data of limited use for epidemiology.

The best sources are therefore research surveys, of which there have been several from different countries in the past 10-20 years. ${ }^{1}$ The problem with these surveys is the variation in the definition of head injury and its severity. Most definitions of head injury depend on evidence of a blow to the head, and exclude facial injuries and foreign bodies in the nose and ears. Scalp, skull, and brain can be injured independently of each other, so that only a proportion of patients with head injuries have initial brain injury. About half of all attenders at accident and emergency departments have a scalp laceration and $15 \%$ have some evidence of brain damage, but only $2 \%$ a skull fracture. ${ }^{2}$ One prominent US epidemiologist suggested that only patients with brain damage should be counted as head injuries - that is, those with some impairment of consciousness either before reaching hospital or on arrival. ${ }^{3}$ But that overlooks the fact that patients with mild injuries without obvious brain damage make a considerable impact on the health care system, both as attenders at accident and emergency departments who are sent home and as admissions. The concern is that a small minority of patients with these mild injuries have serious complications: intracranial haematoma, brain swelling, intracranial infection or epilepsy. They are also important in exploring patterns of causation as the basis for prevention strategies, because most mild injuries might have been more severe with only slightly different circumstances. As for defining severity, most countries use the Glasgow coma scale ${ }^{4}$ : the score can range from 3 to 15 and a convention has emerged that patients with a coma score of 8 or less are classed as severe and those with a score of 13 or more as mild, the others being classed as moderate. Paediatric coma scales have been developed for assessing younger children. ${ }^{5}$

The only systematic data on attenders to accident and emergency departments are from surveys carried out across Scotland in 1974 and 1985, and in one district general hospital in Glasgow in $1984 .^{2}$ These provide a dataset of 12000 attenders, which indicates that about $10 \%$ of all new attenders at accident and emergency departments present with head injuries. Studies have also been done of all admissions and of neurosurgical unit transfers throughout Scotland. ${ }^{6}$ Head injury deaths can be difficult to ascertain from hospital surveys because about half of them occur at the scene and because many fatalities have multiple injuries. $^{7}$

Another source of confusion is the definition of a child. In all our studies we have used under 15 years, but others use under 16 years and some children's hospitals accept only patients of 12 or under. Some studies in other countries classify by decades and record all those under 20 as children. In regard to cause and injury type, complications,
Table 1 Percentage of patients younger than 15 years in different head injury series in Scotland

Accident and emergency department attenders Admissions

Neurosurgical unit transfers

$40-50 \%$

Deaths in neurosurgical unit

Operated intracranial haematoma

$31 \%$

$25 \%$

$20 \%$

$11 \%$

Based on data from Brookes et al. ${ }^{2}$

Table 2 Percentage of people with head injuries presenting to accident and emergency departments with brain damage (Scotland 1985)

\begin{tabular}{lll}
\hline & Children $(n=2118)$ & Adults $(n=3124)$ \\
\hline All & $7 \%$ & $23 \%$ \\
Falls & $6 \%$ & $26 \%$ \\
Road traffic accidents & $27 \%$ & $32 \%$ \\
\hline
\end{tabular}

Based on data from Brookes et al. ${ }^{2}$

and mortality, 15-20 year olds are much more like young adults than children.

The proportion of children in each of the different categories of head injuries varies greatly, ${ }^{2}$ from nearly half of attenders to $20 \%$ of deaths (table 1 ). About half of accident and emergency child attenders are younger than 10 years, but only a fifth of the severely injured and a third of fatalities are patients younger than 5 . Male patients make up more than $70 \%$ of attenders over the age of 5 but only $60 \%$ of the younger children are male.

There are 4011 attenders, 400 admissions, and 5.3 deaths per 100000 children per year. ${ }^{1}$ Age specific rates for deaths and admissions from head injury in the UK across all ages show the peak incidence in the 15-30 age range, but for attenders at accident and emergency departments, this peak is younger than 10 years. ${ }^{1}$ Although the case fatality rate for admitted children is only $0.7 \%$, a fifth of the rate for all ages, the large number of admissions together with the number of deaths before admission means that head injury is an important cause of death in childhood. According to Sharples et $a l^{5}$ head injury is the most common cause of death between 1 and 15 years, accounting for $15 \%$ of all deaths, and for $25 \%$ of deaths in the $5-15$ years age group.

Neuropathologists in Glasgow have compared detailed necropsy findings in 87 children aged 2-15 years with those for 360 adults. ${ }^{8}$ The frequency of contusions, diffuse axonal injury, ischaemic brain damage, and intracranial haematoma was similar in adults and children. However, bilateral cerebral swelling was three to four times more common in children. In $40 \%$ of those the swelling was not accompanied by contusions, ischaemic brain damage or intracranial haematoma - one or more of which are usual in adults with brain swelling.

The vast majority of patients with head injuries coming to accident and emergency departments, and of those admitted, have mild injuries. In the Scottish studies, $80 \%$ of admissions of all ages were for mild injuries, two thirds having neither a skull fracture nor evidence of brain damage, nor an extracranial injury requiring admission in its own right, and two thirds were discharged in less than 48 hours. These admissions reflect extreme caution before guidelines based on statistically calculated risk factors were published (see later).

Of accident and emergency attenders in Scotland, only $1 \%$ of children had impaired consciousness on arrival at hospital, while $6 \%$ had recovered from brief impairment of consciousness. The incidence of brain damage in adults was more than three times that for children, with 5\% impaired on arrival and $18 \%$ recovered from impaired consciousness. In children brain damage was much more common after an RTA than a fall, but no such difference was found in adults (table 2). 
Table 3 Causes of head injury in children in Scotland

\begin{tabular}{llll}
\hline & RTA & Fall & Assault \\
\hline A \& E attenders & $9 \%$ & $57 \%$ & $6 \%$ \\
A \& E attenders with brain damage & $34 \%$ & $45 \%$ & $2 \%$ \\
Admissions & $23 \%$ & $55 \%$ & $5 \%$ \\
Neurosurgical unit transfer & $43 \%$ & $28 \%$ & $4 \%$ \\
Severe injuries & $75 \%$ & $12 \%$ & $3 \%$ \\
Deaths in neurosurgical unit & $71 \%$ & $22 \%$ & $2 \%$
\end{tabular}

Data from Brookes et $a l^{\beta}$ and Jennett et al. ${ }^{9}$

A \& E, accident and emergency department; RTA, road traffic accidents.

Table 4 Causes for accident and emergency department attenders in Scotland

\begin{tabular}{lll}
\hline & Children & Adults \\
\hline Falls & $57 \%$ & $33 \%$ \\
RTA & $9 \%$ & $16 \%$ \\
Assaults & $6 \%$ & $29 \%$ \\
Type of RTA & & \\
Pedestrian & $42 \%$ & $23 \%$ \\
Bicycle & $32 \%$ & $9 \%$ \\
Passenger & $20 \%$ & $58 \%$ \\
\hline
\end{tabular}

Based on data from Brookes et al..$^{2}$

RTA, road traffic accidents.

The distribution of causes of head injury in children varies greatly according to severity, with falls predominant for accident and emergency attenders and admissions, and RTA the major cause only for neurosurgical unit transfers, severe injuries, and deaths (table 3$).{ }^{39}$ For those attending accident and emergency departments, assaults and RTA are more common in adults than in children (table 4). Moreover the distribution of victims of RTA with head injuries are different for children, with fewer car occupants and more pedestrians and cyclists. Among fatal RTAs concerning children, pedestrians are even more common, $69 \%$ in the Newcastle series. ${ }^{10}$ Most bicycle injuries in children are sustained during play off the road and do not involve collision with another vehicle. In some reports these are classified as recreational rather than road accidents. However, most serious injuries are from the minority of bicycle accidents that do involve collisions with other vehicles.

The high incidence of unsafe behaviour by children as a cause of both pedestrian injuries and those caused by bicycle collisions was noted by the Newcastle team. ${ }^{10}$ In that study most child occupants in cars who were fatally injured were not wearing seat belts, and few bicycle fatalities had been wearing helmets. The value of bicycle helmets for children has been studied in Australia ${ }^{11}$ where use is now mandatory in several states. It has been suggested that built-in safety features for restraints in cars should become the rule. ${ }^{12}$ However, most of the recent reduction in child death rates from road accidents in England and Wales appears to be because there are fewer child pedestrians and cyclists as more children travel by car. ${ }^{13}$

Assaults form a small minority of childhood injuries in the UK and are often sustained in play. In the United States, firearms are a frequent cause of injury, mostly the result of children playing with their parents' guns. Even the Newcastle series had four fatal shotgun injuries, all from accidents while playing with parents' guns. ${ }^{10}$ In that study fewer than $5 \%$ of fatal injuries were non-accidental.

A matter of some concern and controversy is the need to admit children with mild injuries to hospital for observation. If unnecessary, this adds unjustifiable stress to the injured child. The main reason for keeping patients under observation is the fear of complications, particularly acute intracranial haematoma. The Newcastle study found one third of avoidable deaths after head injury were because of missed or late diagnosis of this complication. ${ }^{5}$ In 1984 guidelines for the admission of adults with head injuries emphasised the importance of skull fracture and impaired consciousness as risk factors for intracranial haematoma, ${ }^{14}$ and suggested that these criteria should reduce the number of mildly injured patients unnecessarily admitted. More recently, additional data have been published, including those for children, based on the epidemiological studies in Scotland. ${ }^{15}$ These showed that the risk of intracranial haematoma in children is about one sixth of that in adults. However, the risk factors are exactly the same in children, skull fracture being a more potent factor than impaired consciousness. These data indicate the low risk of haematoma in children without skull fracture, only 1 in 500 develop a haematoma even if there is impaired consciousness. Yet in a series of 900 patients in Glasgow operated because of intracranial haematomas there were more children than adults who had been fully conscious, some of them without a skull fracture. Others have questioned the value of skull $x$ ray in assessing head injuries in children. ${ }^{16}$

In Scotland between 1974 and 1985 the admission rate for patients with head injuries for all ages fell from $23 \%$ to $15 \%$, mostly because fewer children were admitted-10\% compared with $20 \%$ of adults in $1985 .^{2}$ Of those with impaired consciousness on arrival at the accident and emergency department, $25 \%$ of children and $16 \%$ of adults were sent home-most often because no skull fracture was found. A skull $x$ ray was done in $58 \%$ of all children, but in $88 \%$ of those with brain damage. Reduction in unnecessary admissions, particularly of children for whom this can add psychological trauma, is to be welcomed. The decision to send a child home will depend on full consciousness having been regained and there being no reason to suspect a skull fracture, but may also be influenced by home circumstances. Moreover, it needs to be associated with adequate counselling of parents about the need for rapid return to hospital in the event of new symptoms, supported by a card listing warning symptoms and giving the telephone numbers for contacting the hospital.

\section{Conclusions}

Head injuries are less common in the UK than in many other countries, but they are still a major problem for health services. Children account for half of attenders at accident and emergency departments after head injury, a third of admissions, a quarter of severe injuries, and a fifth of deaths. Head injuries account for $15 \%$ of deaths of children aged 1-15 years, but the rate is falling because of fewer road accident deaths. Fewer than $10 \%$ of attenders have any evidence of brain damage, and most admissions are patients with mild injuries. Causes vary according to severity, road accidents accounting for fewer than $10 \%$ of attenders but for more than $70 \%$ of severe and fatal injuries. Many injuries on the road are to pedestrians. Fewer children need to be admitted if risk factors for complications in mild injuries are carefully assessed.

B JENNETT

Professor Emeritus, Department of Neurosurgery,

Institute of Neurological Sciences,

Glasgow G51 4TF, UK

1 Jennett B. Epidemiology of head injury. $\mathcal{F}$ Neurol Neurosurg Psychiatry 1996;60:362-9.

2 Brookes M, MacMillan R, Cully S, et al. Head injuries in accident and emergency departments. How different are children from adults? $\mathcal{F}$ Epidemiol Comm Health 1990;44:147-51.

3 Kraus JF, Black MA, Hessol N, et al. The incidence of acute brain injury and serious impairment in a defined population. Am $\mathcal{F}$ Epidemiol 1984;119:186201 .

4 Teasdale G, Jennett B. Assessment of coma and impaired causes: a practical

5 Yager JV, Johnston B, Seishia SS. Coma scales in pediatric practice. Am f Dis Child 1990;144:1088-91.

6 Jennett B, MacMillan R. Epidemiology of head injury. BMF 1981;282: $101-4$.

7 Sharples PM, Storey A, Aynsley-Green A, et al. Avoidable factors contributing to death of children with head injury. BMF 1990;300:87-91. 
8 Graham DI, Ford I, Adams JH, et al. Fatal head injury in children. 7 Clin Pathol 1989;42:18-22.

9 Jennett B, Murray L, Adams JH, et al. Causes of fatal childhood accidents. Jennett B, Murray L,

10 Sharples PM, Storey A, Aynsley-Green, et al. Causes of fatal childhood accidents involving head injury in northern region 1979-86. BMF 1990;301: $1193-7$

11 McDermott F, Love J, Brazenor G, et al. The effectiveness of bicycle helmets in a study of 1710 casualties. F Trauma 1993;34:834-45.

12 Roberts I, Di Guiseppi C. Children in cars. BMF 1997;314:392.
13 Di Giuseppi C, Roberts I, Li L. Influence of changing travel patterns on child death rates from injury: trend analysis. $B M \mathcal{F} 1997 ; 314: 710-13$.

child death rates from injury: trend analysis. BMF 1997;314:710-13. injury in adults. BMF 1984;288:983-89.

15 Teasdale GM, Murray G, Anderson E, et al. Risks of acute traumatic intracranial haematoma in children and adults: implications for managing head injuries. BMF 1990;300:363-7.

16 Lloyd DA, Carty H, Patterson M, et al. Predictive role of skull radiography for intracranial injury in children with blunt head injury. Lancet 1997;349: $821-4$.

\section{Evidence-based dilemmas in pre-school vision screening}

What should policy makers do with systematic reviews that fail to find evidence of effectiveness for interventions currently provided in the NHS? Is no evidence of effectiveness sufficient evidence of no effectiveness? The systematic review process has made great progress in minimising the influence of personal bias in the selection and critical appraisal of evidence. By bringing together in one place and critically appraising all the relevant studies, these reviews can show the fragility of the evidence on which some current practice is based.

Policy makers in the NHS take the results of these reviews seriously and expect to be able to act on their findings. But clinicians argue that it is wrong to close down a service, which might be doing good, just because the research evidence of effectiveness is poor. The review of pre-school vision screening commissioned by the NHS Health Technology Assessment Programme ${ }^{1}$ raises this dilemma and presents policy makers with a difficult decision. This report's most startling finding was that the reviewers could not identify any robust studies showing that amblyopia (the main target condition of pre-school vision screening programmes ${ }^{2}$ ) causes any problems to children or adults. One researcher who tried to find performance differences between amblyopic students (as opposed to people blinded in one eye in adulthood or blindfolded in one eye for the purpose of an experiment) and students with monocular refractive errors was not able to document disabilities that he considered likely to affect everyday life. ${ }^{3}$ Health professionals have always assumed that reduced vision in one eye must cause problems, and in occupational health services health professionals have been responsible for the development of policies that exclude people with amblyopia from certain occupations on these grounds. The best documented consequence of amblyopia, however, appears to be that blindness may ensue if the good eye becomes damaged or diseased in later life. The incidence of this problem has been reported in a single study from Finland, ${ }^{4}$ which was not methodologically robust. The problem is certainly rare compared with childhood amblyopia, which has a prevalence of $1-2 \%$ and is unlikely to be sufficiently common to justify treating all childhood amblyopes. Surprised by these findings, the authors of the review went on to undertake a qualitative study that suggested that people who have suffered from amblyopia since childhood have difficulty in specifying any way this has affected them. It may, however, be difficult for people to perceive that they cannot perceive something of which they have no experience.

The second finding of the review was that it was not possible to identify trials of treatment for amblyopia that included a "no treatment" control group. The natural history of amblyopia has only been studied in an indirect way and for short periods, and the studies that have been done suggest that amblyopia may sometimes regress spontaneously. Given the lack of studies on the natural history of the condition, the results of observational studies of treatment impact (which show an average improvement of around two lines on a Snellen chart) and studies comparing one treatment regimen with another, cannot be interpreted with any confidence. The evidence for clinicians' belief in the efficacy of amblyopia treatment therefore comes largely from animal studies in which amblyopia has been artificially created in kittens and monkeys. The pharmaceutical industry would never be allowed to market a drug that had not been thoroughly tested in humans. It seems unlikely that the public would, if they knew, approve of other types of interventions being done on their children on the basis of animal studies alone.

An incidental finding of the qualitative study was that many adults and children were clear that the treatment (wearing glasses and intermittent patching of the good eye) had had an important and negative impact on their quality of life as children and on the lives of their families. ${ }^{5}$ The children did not like wearing glasses or patches, and some parents had felt distressed at enforcing these on their children. They persisted for two reasons, however. Firstly, because they were concerned that if they did not their children's sight might cause problems in later life and secondly, because they felt that good parents should do for their children what was prescribed by health professionals.

The review found one prospective controlled study and a small number of retrospective observational studies that confirmed beliefs that primary orthoptic screening is an efficient way of identifying children with this condition. In the face of the evidence that the condition may not be disabling, that there are no robust studies of treatment impact, and that the psychological and practical consequences of treatment could be a problem for families, this evidence may be redundant. This review set out to investigate the efficacy of screening and therefore concluded that screening is not effective, not because the screening programme cannot identify children efficiently, but because there is no evidence that treatment is either effective or necessary. It has therefore called into question the provision of orthoptic services as well as screening services and has presented policy makers with an added dilemma.

Although amblyopia is regarded as the main target condition for this programme, pre-school vision screening also identifies other conditions that are sufficiently common to warrant screening, for example, non-cosmetically obvious squints (phorias and microsquints) and refractive errors. Cosmetically disturbing squints are by definition obvious to the casual observer and therefore do not need to be screened for. Spectacle correction is effective in correcting refractive errors. There is, however, no clear evidence that pre-school children benefit from correction of the minor refractive errors that are common in childhood, and there is good evidence that children with these defects, who are old enough to make choices for themselves, often choose not to wear glasses. ${ }^{6}$ Glasses are sometimes prescribed to 
children with refractive errors to prevent the development of amblyopia or squint, but the reviewers found no controlled studies of any sort showing that this was effective at the age of 3 years. Phorias and microsquints are only treated if there is accompanying amblyopia or refractive error; if not, they are watched so that if the squint progresses an operation can be undertaken.

It may be that in the future more detailed studies will define and quantify difficulties suffered by people with amblyopia and other common childhood visual problems, and that properly conducted randomised controlled trials of treatment will show that these difficulties can be reduced by intervention. Such studies may alternatively show that the difficulties are indeed insignificant and that the detrimental impact of intervention on families and children is significant and important. In the meantime, the NHS is supporting an intervention for which there is no evidence base. This intervention consumes scarce resources, but more importantly it requires parents to enforce a potentially distressing treatment on their children, on specious premises.

The results of this report suggest that health authorities should stop purchasing pre-school vision screening services. An invitation for screening carries with it the implicit assumption that children will benefit from being identified and it is therefore unethical to screen in the absence of robust evidence of treatment impact. ${ }^{7}$ In the case of screening, "no evidence of effectiveness is sufficient evidence of no effectiveness." The situation with regard to treatment services is slightly different. It is regarded as ethical for a clinician to treat someone to the best of his ability on the basis of his clinical experience. However, because amblyopia is asymptomatic, children with amblyo- pia rarely present spontaneously unless they also have another problem.

A decision to stop purchasing orthoptic services for the treatment of amblyopia would have serious consequences for orthoptists. Such a decision might also be difficult for parents, teachers, and other members of the public to accept after they have been told for many years how important it is to treat children with these problems. It would result in the dismantling of a service that could not be re-established quickly overnight. If policy makers do not feel able to take this step, a reasonable and ethical compromise could be that they insist that treatment be offered only after a clear statement of the limits of medical knowledge about the conditions, their treatment, and their side effects. Then parents could make up their own minds about whether the potential benefits are worth the problems that treatment causes.

SARAH L STEWART-BROWN SARAH K SNOWDON

Health Services Research Unit,

Department of Public Health,

University of Oxford, Oxford OX3 7LF, UK

1 Snowdon SK, Stewart-Brown SL. Preschool vision screening: results of a systematic review. York: Centre for Reviews and Dissemination, 1997. 2 Hall DMB. Health for all children. Oxford: Oxford University Press, 1996

3 Kani W. Human amblyopia and its perceptual consequences. PhD thesis. Kani W. Human amblyopia and its perce
Durham: University of Durham, 1980.

4 Tomila V, Tarkanen A. Incidence of loss of vision in the healthy eye in amblyopia. Br f Ophthalmol 1981;65:575-7

5 Snowdon SK, Stewart-Brown SL. Amblyopia and disability: a qualitative study. Oxford: University of Oxford, 1997.

6 Stewart-Brown SL. Spectacle prescribing amongst ten year old children. $\mathrm{Br}$ f Ophthalmol 1985;69:874-80.

7 Mant D, Fowler G. Mass screening: theory and ethics. BMf 1990;300: 916-18. 